\title{
cGAS/STING axis mediates a topoisomerase II inhibitor-induced tumor immunogenicity
}

\author{
Zining Wang, ${ }^{1}$ Jiemin Chen, ${ }^{1}$ Jie Hu, ${ }^{2}$ Hongxia Zhang, ${ }^{1}$ Feifei Xu, ${ }^{1}$ Wenzhuo He, ${ }^{1}$ Xiaojuan Wang, ${ }^{1}$ Mengyun Li, ${ }^{1,3}$ Wenhua Lu, ${ }^{1}$ \\ Gucheng Zeng, ${ }^{4}$ Penghui Zhou, ${ }^{1}$ Peng Huang, ${ }^{1}$ Siyu Chen, ${ }^{5}$ Wende Li, ${ }^{5}$ Liang-ping Xia, ${ }^{1,6}$ and Xiaojun Xia ${ }^{1}$ \\ 'State Key Laboratory of Oncology in South China, Collaborative Innovation Center for Cancer Medicine, Sun Yat-sen University Cancer Center, Guangzhou, China. ${ }^{2}$ Department of Medical Oncology, The First \\ Affiliated Hospital of Sun Yat-sen University, Guangzhou, China. ${ }^{3}$ College of Food Science and Engineering, Northwest A\&F University, Yangling, China. ${ }^{4}$ Department of Microbiology, Zhongshan School \\ of Medicine, Key Laboratory for Tropical Diseases Control of the Ministry of Education, Sun Yat-sen University, Guangzhou, China. ${ }^{5}$ Guangdong Laboratory Animals Monitoring Institute, Guangdong Key \\ Laboratory of Laboratory Animals, Guangzhou, China. ${ }^{6}$ Department of Medical Oncology, Sun Yat-Sen University Cancer Center, Guangzhou, China.
}

\begin{abstract}
Checkpoint blockade antibodies have been approved as immunotherapy for multiple types of cancer, but the response rate and efficacy are still limited. There are few immunogenic cell death-inducing (ICD-inducing) drugs available that can kill cancer cells, enhance tumor immunogenicity, increase in vivo immune infiltration, and thereby boost a tumor response to immunotherapy. So far, the ICD markers have been identified as the few immunostimulating characteristics of dead cells, but whether the presence of such ICD markers on tumor cells translates into enhanced antitumor immunity in vivo is still being investigated. To identify anticancer drugs that could induce tumor cell death and boost T cell response, we performed drug screenings based on both an ICD reporter assay and a T cell activation assay. We showed that teniposide, a DNA topoisomerase II inhibitor, could induce high-mobility group box 1 (HMCB1) release and type I IFN signaling in tumor cells and that teniposide-treated tumor cells could activate antitumor T cell response both in vitro and in vivo. Mechanistically, teniposide induced tumor cell DNA damage and innate immune signaling, including NF-кB activation and stimulator of IFN genes-dependent (STING-dependent) type I IFN signaling, both of which contribute to the activation of dendritic cells and subsequent T cells. Furthermore, teniposide potentiated the antitumor efficacy of anti-PD1 in multiple types of mouse tumor models. Our findings showed that teniposide could trigger tumor immunogenicity and enabled a potential chemoimmunotherapeutic approach to potentiating the therapeutic efficacy of anti-PD1 immunotherapy.
\end{abstract}

\section{Introduction}

Cancer immunotherapy has become the major theme of cancer treatment regimens in the last few years, testifying to the genuine capability of the immune control of cancer $(1,2)$. Among the few highly successful immunotherapeutic approaches, antiPD1 antibody has demonstrated impressive efficacy across several different cancer types $(3,4)$. Mechanistically, anti-PD1 or anti-PD-L1 blocking antibodies block the interaction between PD-L1/L2 and PD1 receptor and the immunosuppressive signal on $\mathrm{T}$ cells, thus restoring the antitumor function of exhausted $\mathrm{T}$ cells (5-7). Despite the prevalent success in different types of cancers, the response rate of anti-PD1 antibody therapy is quite low, ranging from $20 \%$ to $40 \%$, and reliable biomarkers for predicting therapeutic response or efficacy are still lacking $(2,3)$. Accumulating evidence suggests that the tumor response to anti-PD1 is highly dependent on tumor immunogenicity (i.e., tumor mutation burden, neoantigen abundance), intratumor PD-L1 expression, and an immune-active tumor microenviron-

Conflict of interest: The authors have declared that no conflict of interest exists. Copyright: () 2019, American Society for Clinical Investigation.

Submitted: January 15, 2019; Accepted: August 7, 2019; Published: October 7, 2019

Reference information: J Clin Invest. 2019;129(11):4850-4862.

https://doi.org/10.1172/JCI127471. ment (8-11). Immunogenic cell death-inducing (ICD-inducing) drugs may enhance tumor antigen exposure, boost the release of immune-stimulating tumor cell content, and elicit immune cell infiltration, thus converting the immune "cold" tumor into "hot" tumor. The combination of such drugs with immunotherapy, i.e., anti-PD1, may enhance the antitumor efficacy and expand the benefit of immunotherapy $(12,13)$. Thus, identifying drugs that can both enhance tumor immunogenicity and potentiate tumor response to anti-PD1 therapy is needed.

Recent studies have shown that some chemotherapeutic drugs could induce tumor cell ICD, which could potentially elicit or enhance an antitumor immune response (13-17). The common features of ICD are upregulated expression or release of damage-associated molecular patterns (DAMPs) by the dying tumor cells (13). The release of high-mobility group box 1 (HMGB1) and ATP could serve as chemoattractant signals to recruit the antigen-presenting cells (APCs), such as DCs and macrophages. In addition, the membrane translocation of calreticulin (CRT) on the dying tumor cells serves as "eat me" signaling to promote phagocytosis or efferocytosis by the APCs, which process and present the tumor antigens to $\mathrm{T}$ cells $(18,19)$. Also, innate immune signaling activation, such as that by the NF- $\mathrm{\kappa B}$ pathway, and IFN-I signaling activation in dying tumor cells induce inflammatory cytokines, chemokines, and IFN-I production, which in turn promote 
A
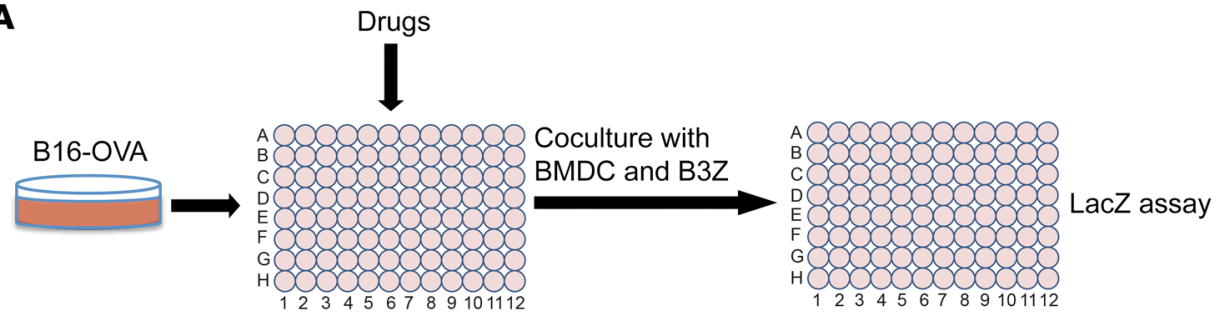

B

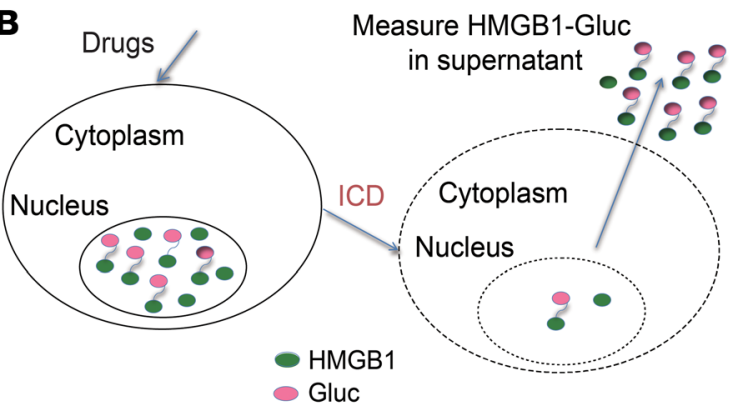

D
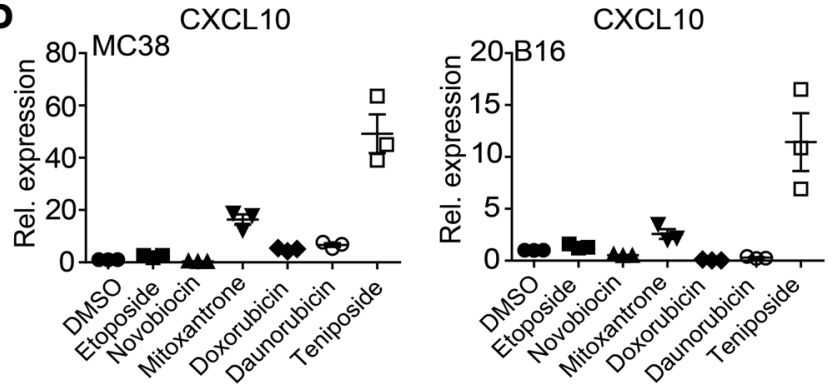

DC maturation and T cell activation $(13,20,21)$. Accordingly, the dying tumor cells work as an in situ vaccine by attracting APCs and inducing their activation and maturation to present tumor antigens to $\mathrm{T}$ cells, which subsequently undergo proliferation and attack tumor cells specifically (13). In this way, ICD-inducing chemotherapy drugs not only kill cancer cells, but also activate anticancer immunity that may potentiate the therapeutic efficacy of immunotherapy (22). However, all the known ICD markers do not always translate into strong in vivo antitumor immunity, and systemic evaluation of the ICD features of currently approved drugs have yielded inconsistent results due to tumor-intrinsic variation of capacities of some cellular functions such as autophagy or necroptosis $(19,23,24)$.

Currently, there are several reporter systems used for ICD inducer drug screening, such as fluorescent biosensor of CRTGFP or HMGB1-GFP, ATP, and ELISA measurement of HMGB1 (25). As cells undergo ICD, CRT will translocate from the perinuclear ER to the cell surface, and HMGB1 will be released from the nucleus to the extracellular space. Such intracellular translocation can be tracked by the fused GFP fluorescent signal change that can be detected with fluorescent microscopy (17). However, ICD features cannot be reflected by a single marker; therefore, positive hits from one assay may not convincingly enhance tumor immunogenicity. Furthermore, the ICD phenotype of tumor cells may not always induce immune cell activation. To accurately measure drug-induced tumor immunogenicity, we
Figure 1. T cell-based drug screening identified ICD inducers. (A) Outline of drug-screening protocol. B16-OVA tumor cells were seeded on 96-well plates and treated with drugs for 16 hours, then cocultured with BMDC and B3Z cells for 24 hours. LacZ reporter activity was measured as a surrogate marker for T cell activation. (B) Illustration of the principle of the HMCB1-Gluc reporter system. Once drugs or inhibitors induce tumor cell ICD, HMCB1-Gluc is released from the nucleus into the supernatant, and supernatant luciferase activity is detected. (C) MC38 (HMCB1-Gluc) cells were treated with different Top inhibitors or DMSO for 20 hours; then HMGB1-Gluc luciferase (Gluc luc) activity was measured. (D) MC38 and B16 cells were treated as in C, and then the mRNA expression level of CXCL10 was measured by qPCR. Rel., relative. Data in $\mathbf{C}$ and $\mathbf{D}$ are shown as mean \pm SD of 3 independent experiments. designed a T cell activation assay as well as an HMGB1-Gaussia luciferase (HMGB1-Gluc) reporter assay. In the T cell activation assay, $T$ cells and DCs were cocultured with tumor cells pretreated with different drugs, and then $\mathrm{T}$ cell activation was measured by detecting extracellular IL-2 levels (detected by ELISA) or IL-2 promoter-driven $\beta$-galactosidase (LacZ) reporter gene activity. In the HMGB1-Gluc assay, the HMGB1 release, one hallmark of ICD, was detected by measuring HMGB1-fused Gluc activity. By combining 2 screening assay results, we showed that the DNA topoisomerase II (Top II) inhibitor teniposide was capable of eliciting tumor cell ICD and subsequent T cell activation. Further investigations revealed that teniposide could induce DCs and $\mathrm{T}$ cell activation and protect against tumor growth when used in a vaccine setting. Teniposide could also upregulate the antigen presentation machinery on tumor cells. More importantly, teniposide induced NF- $\mathrm{KB}$ and type I IFN pathway activation through the cyclic GMP-AMP synthase/stimulator of IFN gene (cGAS/STING) axis and potentiated DC-mediated antigen presentation to $\mathrm{T}$ cells. On both immunotherapy-sensitive and -resistant mouse tumor models, teniposide promoted the tumor infiltration and activation of $\mathrm{CD} 8^{+} \mathrm{T}$ cells and boosted the antitumor efficacy of anti-PD1 therapy. Our findings suggest that teniposide could increase tumor immunogenicity, boost antitumor immunity, and provide a potential chemoimmunotherapeutic approach for cancer treatment by teniposide in combination with anti-PD1 antibody. 


\section{Table 1. Drugs that elicited highest LacZ activity and HMGB1-Gluc activity}

\begin{tabular}{lccc} 
Drugs & Bioactivity & $\begin{array}{c}\text { LacZ activity } \\
\left(\mathbf{O D}_{590}\right)\end{array}$ & $\begin{array}{c}\text { HMGB1-Cluc activity } \\
(\text { fold })\end{array}$ \\
Acrisorcin & Antifungal & 2.21 & 11.5 \\
Teniposide & Antineoplastic & 2.12 & 5.97 \\
Prednisolone tebutate & Antiinflammatory & 2.06 & 1.44 \\
Algestone acetophenide & Antiacne & 1.82 & 1.38 \\
Methscopolamine bromide & Anticholinergic & 1.81 & 1.15 \\
Flurandrenolide & Antiinflammatory & 0.93 & 0.86 \\
\hline
\end{tabular}

\section{Results}

Combined drug-screening assays identified teniposide as an ICD drug. ICD drug screening has been reported by several groups, but so far, the known ICD markers cannot directly reflect the $\mathrm{T}$ cell activation induced by dead tumor cells. To circumvent this issue, we adapted an antigen presentation assay to directly examine $\mathrm{T}$ cell activation upon tumor cell death. We treated OVA-expressing B16 mouse melanoma cells (B16-OVA) with an FDA-approved drug library for 16 hours, cocultured with bone marrow-derived DCs (BMDCs) and OVA-specific CD8 ${ }^{+} \mathrm{T}$ cell hybridoma B3Z cells for 24 hours, then measured IL-2 promoter-driven LacZ activity, which reflected IL-2 expression (Figure 1A). Among the 1280 drugs tested, we identified a few drugs that could activate LacZ activity in B3Z cells in this assay (Table 1). To corroborate the results, we also designed an HMGB1-Gluc reporter assay to screen for drugs that could elicit the release of HMGB1, which is one of the hallmarks of ICD. In this HMGB1-Gluc reporter assay, HMGB1 was fused with a Gluc, and drug-induced HMGB1-Gluc release elicited luciferase activation (Figure 1B and Supplemental Figure 1, A-C; supplemental material available online with this article; https://doi.org/10.1172/JCI127471DS1). In this reporter assay, acrisorcin and teniposide induced the highest level of HMGB1-Gluc activation above the basal level (Table 1). As teniposide was the only FDA-approved antineoplastic drug that we identified from both screening assays, we chose to focus on this drug for the subsequent experiments.

Teniposide is a Top II inhibitor currently used for treating several types of cancer, including childhood acute lymphocytic leukemia. Interestingly, a few Top II inhibitors, such as mitoxantrone and doxorubicin, have been previously identified as a prototype of drugs eliciting cancer cell ICD features (18). We therefore compared the capabilities of these topoisomerase inhibitors for activating HMGB1 release. Among the 6 inhibitors tested, teniposide elicited the highest HMGB1-luc activity (Figure 1C). As drug-induced chemokine CXCL10 release has been recently considered as a new ICD marker, we measured CXCL10 expression in cancer cells after treatment with the topoisomerase inhibitors. Consistently, teniposide was found to be able to induce the highest CXCL10 expression in both B16 mouse melanoma cells and MC38 mouse colon cancer cells (Figure 1D).

Teniposide induces tumor cell ICD. The ability of teniposide to stimulate cancer cell ICD was then investigated. Teniposide treatment induced HMGB1-luc activity in a dose-dependent fashion
(Figure 2A and Supplemental Figure 2, A and B). Moreover, it also induced tumor cell death, which was detected by flow cytometry, lactate dehydrogenase (LDH) release (Figure 2B and Supplemental Figure 2, C and D), and surface expression of CRT, another known ICD marker (Figure 2C and Supplemental Figure 2E). When mice bearing CT26 tumors were treated with teniposide, CRT expression levels in tumor tissues also increased (Supplemental Figure $2 \mathrm{~F}$ ). To unambiguously validate the in vivo effect of the tumor immunogenicity elicited by teniposide, we examined the immunogenicity of teniposide-treated tumor cells in a vaccination setting (26). We treated CT26 tumor cells with teniposide in vitro and injected the dead cells into the left flank of immunocompetent BALB/c mice. The mice were then rechallenged with live CT26 cell inoculation into the right flank 8 days later. We observed $100 \%$ tumor-free survival among mice immunized with teniposide-treated dead tumor cells in the 30 days after challenge, while all mice that were vaccinated with freeze-thawed tumor cells developed tumors (Figure 2D). As a comparison, vaccination with tumor cells pretreated by another Top II inhibitor, etoposide, only showed partial and temporary protection against tumor growth from live tumor cell rechallenge in such a setting. These results together confirmed teniposide as a bona fide ICD inducer.

Teniposide upregulated expression of tumor cell antigen presentation machinery. As tumor antigen expression on the tumor cell surface is essential for $\mathrm{T}$ cell recognition and killing, we investigated the influence of teniposide on the expression of tumor antigen presentation machinery components. Teniposide treatment increased MHC-I and MHC-II expression on the tumor cell surface (Figure 3, A and B). Specifically, genes encoding mouse $\beta 2 \mathrm{~m}(\mathrm{~B} 2 \mathrm{~m})$, an essential component of MHC-I, were upregulated in teniposide-treated tumor cells, as were the genes directing peptide cleavage (Erap1), peptide transporters (Tap1 and Tap2), and transporter-MHC interactions (Tapbp) (Figure 3C). Furthermore, teniposide treatment increased the surface expression of MHC-I-bound SIINFEKL (OVA epitope peptide) complex on OVA-expressing mouse tumor cell lines (B16-OVA and MC38OVA) (Supplemental Figure 3A). Ex vivo analysis of CT26 tumors also verified increased levels of MHC-I, MHC-II, and antigen presentation machinery gene expression after teniposide treatment (Supplemental Figure 3B). Taking these data together, teniposide was found to have the potential to enhance the expression of tumor antigen presentation machinery molecules.

Tumor cells treated with teniposide induce $T$ cell activation and DC activation. We next determined the activation of T cells and DCs when they were cocultured with teniposide-treated tumor cells. We treated B16-OVA cells with DMSO vehicle or teniposide for 20 hours, then cocultured them with BMDCs and B3Z T cells for 24 hours. Consistent with the increased LacZ activity (Figure 4A), the supernatant levels of T cell-derived cytokines IL- 2 and IFN- $\gamma$ significantly increased in $\mathrm{T}$ cells cocultured with tumor cells pretreated with teniposide (Figure 4, B and C). Meanwhile, the proportion of $\mathrm{T}$ cells expressing the activation marker CD69 and effector molecule granzyme B (Gzm B) also increased after coculture (Figure 4D and Supplemental Figure 4A). Similar results were obtained when primary OT-I T cells were used instead of B3Z cells (Figure 4, E-G, and Supplemental Figure $4 \mathrm{~B})$. Collectively, these data demonstrate that teniposide could boost $\mathrm{T}$ cell activation. As DCs play a key role in the recognition of DAMPs 
A

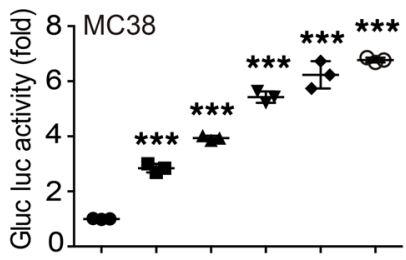

Teniposide $(\mu \mathrm{M}) 0 \quad 0.6 \quad 1.2 \quad 2.5 \quad 5 \quad 10$

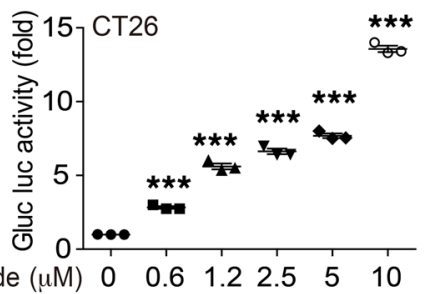

Teniposide $(\mu \mathrm{M}) \quad 0 \quad 0.6 \quad 1.22 .5 \quad 5 \quad 10$
B

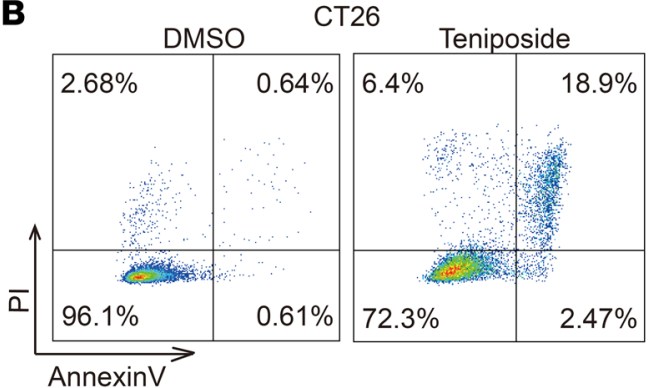

C

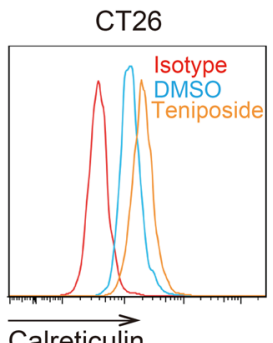

\section{CT26}

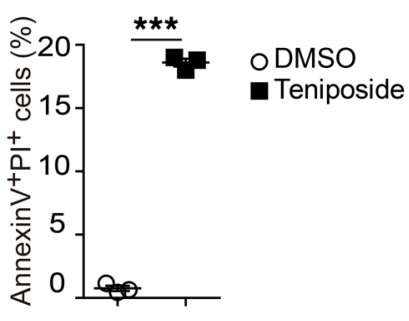

Figure 2. Teniposide induced ICD of tumor cells. (A) MC38 (HMGB1-Gluc) and CT26 (HMGB1-Gluc) cells were treated with increasing doses of teniposide for 20 hours, and HMCB1-Gluc luciferase activity was measured. (B and C) CT26 cells were treated with teniposide or DMSO for 20 hours, and cell apoptosis (B) and surface expression of CRT(C) were detected by FACS. (D) CT26 tumor cells were pretreated with teniposide, etoposide, or freeze-thawed, followed by subcutaneous inoculation into BALB/c mice as a vaccine ( $n=8$ for control group with no tumor cell vaccine administered, teniposide group, and freeze-thawed group; $n=5$ for etoposide group). After 8 days, mice were rechallenged with live CT26 cells. Shown are the percentages of tumor-free mice $\mathbf{3 0}$ days after rechallenge. Data in $\mathbf{A}-\mathbf{C}$ are shown as mean \pm SD of 3 independent experiments. ${ }^{* *} P<0.01$; ${ }^{* *} P<0.001$, 1-way ANOVA with Bonferroni's post test (A), unpaired Student's $t$ test (B), log-rank (Mantel-Cox) test (D).

Calreticulin

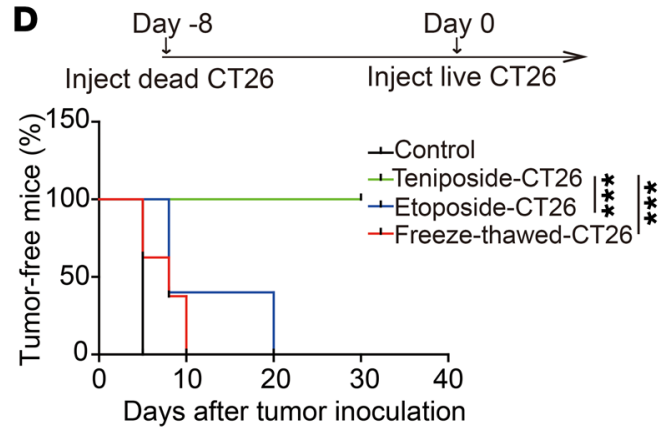

associated with ICD and the subsequent uptake and presentation of tumor antigens to $\mathrm{T}$ cells, we next examined the activation status of DCs cocultured with teniposide-treated tumor cells. Teniposide-treated B16 or MC38 tumor cell coculture markedly increased the surface expression of activation markers, including CD80, CD86, MHC-I, MHC-II, and CD40 on BMDCs (Figure 4, H-L, and Supplemental Figure 4C). Moreover, the surface expression level of MHC-I-bound SIINFEKL complex also significantly increased (Figure 4M). These data showed that teniposide-killed tumor cells induced BMDC maturation, antigen presentation, and subsequent $\mathrm{T}$ cell activation.

Teniposide induces tumor cell immunogenicity by activating $N F-\kappa B$ and type I IFN signaling. Recent studies have shown that some types of chemotherapy or irradiation could induce tumor cell DNA damage, cell death, and inflammatory response, which could subsequently activate antitumor immunity, depending on the specific context $(12,27,28)$. DNA damage marker $\gamma-\mathrm{H} 2 \mathrm{AX}$ expression was detected in tumor cells after teniposide treatment (Figure 5A and Supplemental Figure 5A). Consistently, genomic DNA was detected in cytoplasm after teniposide treatment (Supplemental Figure 5B). Interestingly, teniposide induced the highest levels of genomic DNA leaked in cytoplasm among the few inhibitors tested. At the molecular level, both phosphorylated and total STAT1 protein levels were found to increase after teniposide treatment (Supplemental Figure 5C), indicating IFN-I signaling activation. Interestingly, cGAS protein levels also increased after teniposide treatment (Supplemental Figure 5D). In addition,
$\mathrm{NF}-\kappa \mathrm{B}$ signaling was also activated, as evidenced by increased levels of p65 phosphorylation (Supplemental Figure 6A). As a consequence of the IFN-I and NF- $\kappa$ B activation, significant increases in mRNA and protein levels of downstream cytokines CCL5 and CXCL10 were detected in B16, CT26, and MC38 cells and in tumor tissues after teniposide treatment (Figure 5, B and C, and Supplemental Figure 5, E-G).

The cGAS/STING pathway has been previously shown to be able to sense and respond to cytoplasmic DNA or micronuclei, both of which are indicative of damaged DNA that has escaped the nucleus $(29,30)$. To investigate the role of this pathway in IFN-I activation, we generated Sting ${ }^{-/-}$B16 and MC38 cell lines using the clustered regularly interspaced short palindromic repeats (CRISPR)/Cas9 gene-KO technique and confirmed that STING protein expression was absent in these cells (Supplemental Figure 5I). The KO of STING abolished teniposide-induced IFN-I pathway activation (Figure 5D and Supplemental Figure $5 \mathrm{H})$. More importantly, IL-2 production and CD69 expression on $\mathrm{T}$ cells were markedly attenuated when cells were cocultured with teniposide-treated $\mathrm{Cgas}^{-/-}$and Sting ${ }^{-/}$B16-OVA cells as compared with $\mathrm{T}$ cells cocultured with teniposide-treated WT B16OVA cells (Figure 5, E and F). Interestingly, tumor cells pretreated with IKK inhibitor BAY-117082 or transduced with shRNA targeting IKK $\beta$ also induced significantly lower levels of $T$ cell activation upon teniposide treatment (Supplemental Figure 6, B-D). Knockdown of TBK1 or IRF3 expression by gene-specific shRNAs partially inhibited $\mathrm{T}$ cell activation (Supplemental Figure 6, E and 
A

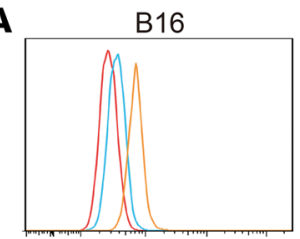
MHC-I

B
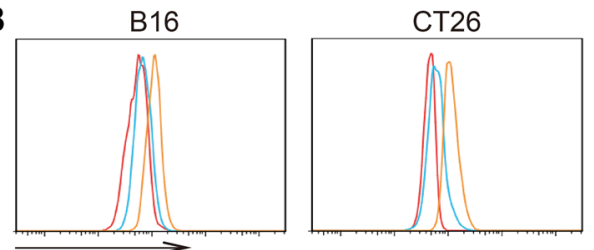

MHC-II

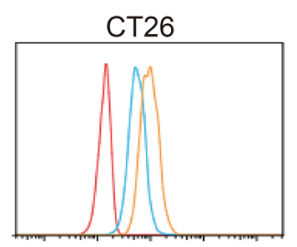

CT26
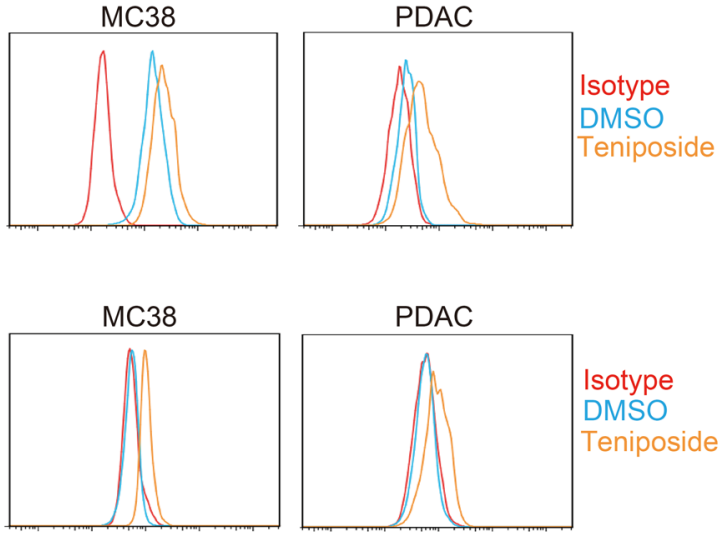

C
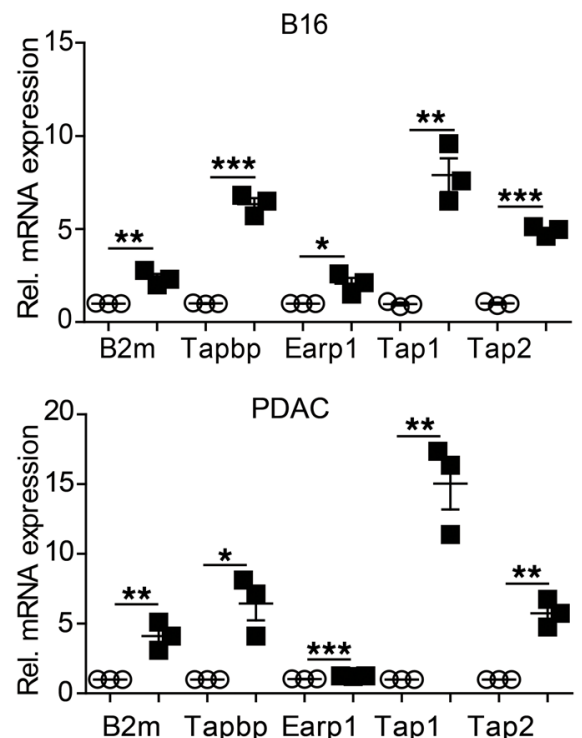

B2m Tapbp Earp1 Tap1 Tap2
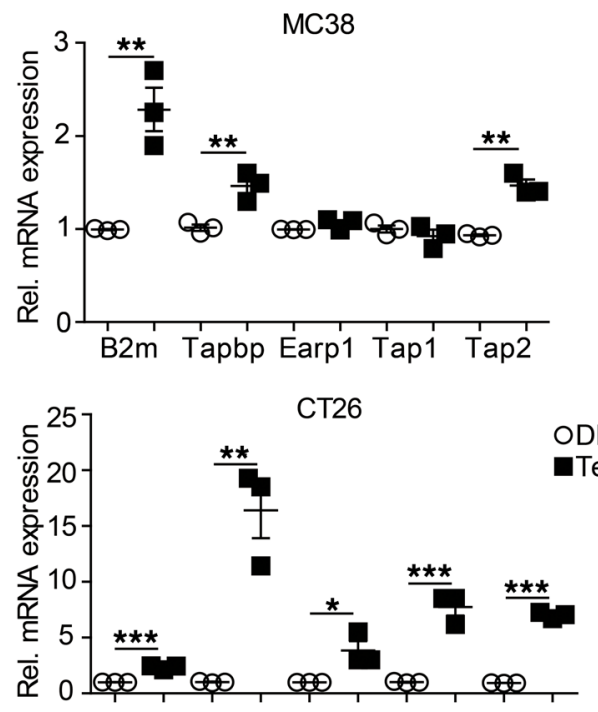

B2m Tapbp Earp1 Tap1 Tap2

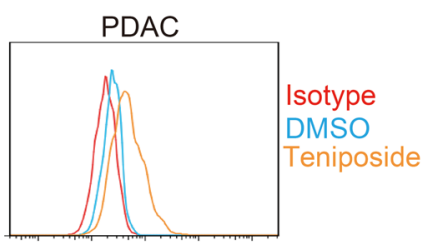

Figure 3. Teniposide enhanced expression of antigen-presenting machinery molecules on tumor cells. (A and B) B16, MC38, PDAC, and CT26 cells were treated with teniposide or DMSO for 20 hours, and the surface expression of MHC-I and MHC-II was determined by FACS. (C) Cells were treated as in $\mathbf{A}$, and the expression of antigen-presenting machinery genes were measured by qPCR. Data in $\mathbf{A}$ and $\mathbf{B}$ are shown as the representative results of 3 repeated experiments. Data in C are shown as mean \pm SD of 3 independent experiments. ${ }^{*} P<$ 0.05 ; ${ }^{*} P<0.01$; ${ }^{* * *} P<0.001$, unpaired Student's $t$ test.
F). In comparison, the pretreatment of tumor cells with inhibitors blocking RIPK1 (Nec-1), JNK (SP600125), ROS (NAC), or caspase (zVAD-FMK) did not have any effect on T cell activation (Supplemental Figure 6G). Thus, both NF- $\kappa$ B and cGAS/STING signaling are required for teniposide-induced tumor immunogenicity. To confirm that IFN-I activation in tumor cells could contribute to the DC function, we then treated B16-OVA cells with teniposide and cocultured them with WT or Ifnar-1- BMDCs together with B3Z cells. We observed significantly attenuated LacZ activation and IL-2 and IFN- $\gamma$ secretion in B3Z cells cocultured with Ifnar-/ BMDCs (Figure 5, G-I). These results together suggest that teniposide could induce NF- $\kappa \mathrm{B}$ and cGAS/STING pathwaydependent IFN-I signaling activation in tumor cells, which in turn activate DCs and T cells.

Teniposide sensitizes tumor response to anti-PD1 treatment. Our observations indicated that teniposide could enhance the immunogenicity of tumor cells, which prompted us to examine the impact of teniposide treatment on the tumor microenvironment. Treatment with teniposide demonstrated significant tumor growth inhibition in CT26 and B16 tumor models (Figure 6, A and B, and Supplemental Figure 7A). Using flow cytometry analysis of single cells isolated from tumor tissues, we found that teniposide treatment increased the percentage of tumor-infiltrating $\mathrm{T}$ cells and the number of tumor-infiltrating $\mathrm{CD} 8^{+} \mathrm{T}$ cells, but not that of $\mathrm{CD}^{+}{ }^{+} \mathrm{T}$ cells (Figure 6, C-E). A similar trend was observed, as $\mathrm{CD}^{+}$ $\mathrm{T}$ cell infiltration in $\mathrm{B} 16, \mathrm{MC} 38$, and the pancreatic ductal adenocarcinoma (PDAC) pancreatic tumor model was found to increase (Supplemental Figure 7, A and B). Moreover, a higher proportion of tumor-infiltrating $\mathrm{T}$ cells in the treatment group expressed the T cell activation marker CD69 and effector molecules Gzm B and IFN- $\gamma$, but not TNF- $\alpha$, as compared with the control group (Figure 6, F-J, and Supplemental Figure 7C). Tumor tissue-derived DCs showed increased levels of MHC-I, MHC-II, CD40, and CD86 after teniposide treatment (Figure 6, K-N). Importantly, CD8 ${ }^{+} \mathrm{T}$ cells are required for antitumor efficacy of teniposide, as pretreatment with anti-CD8 depletion antibody, but not anti-CD4 depletion antibody, abolished teniposide-induced CT26 tumor inhibition in BALB/c mice (Figure 6O).

Interestingly, teniposide also increased the PD-L1 surface expression on tumor cells (Supplemental Figure 7D). As intratumor PD-L1 expression and T cell infiltration are the 2 major hallmarks of tumors responding to anti-PD1 therapy in the clinic (3), we next tested the therapeutic efficacy of combining teniposide with anti-PD-1 treatment in the CT26 tumor model, which 
A

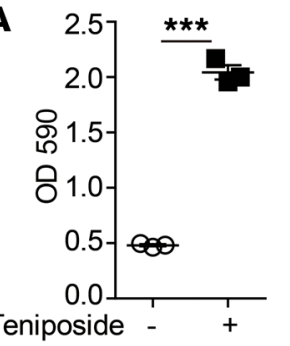

E

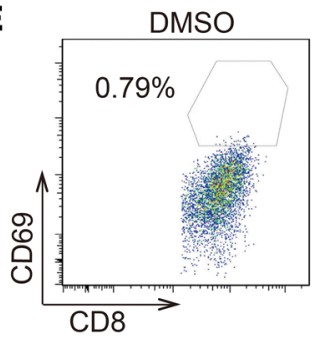

G

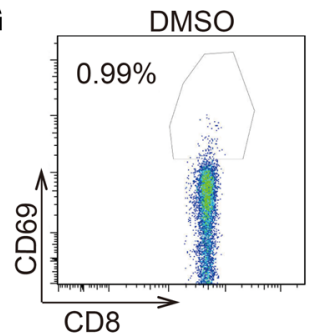

H

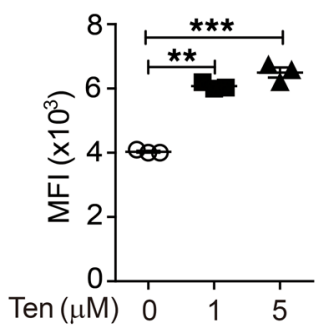

$\mathbf{K}$

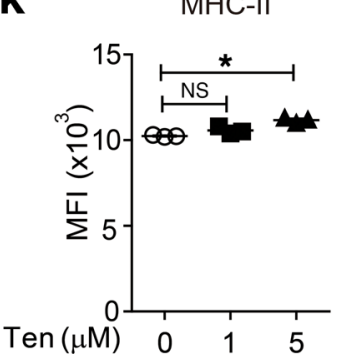

B
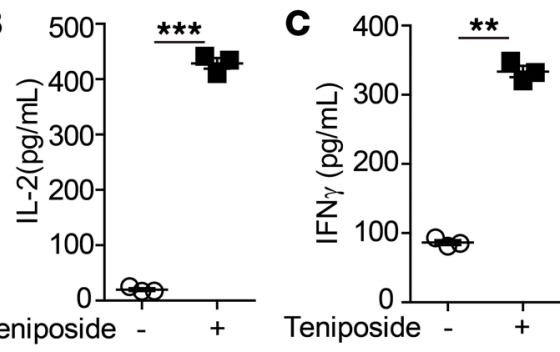

Teniposide
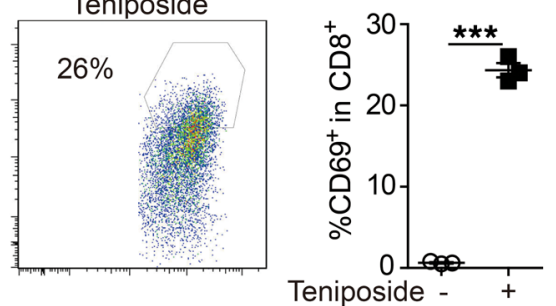

Teniposide

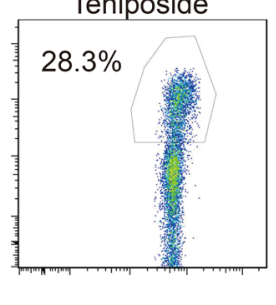

I

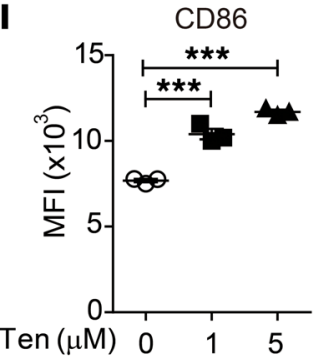

$\mathbf{L}$

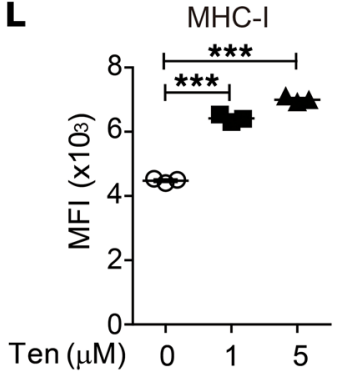

D

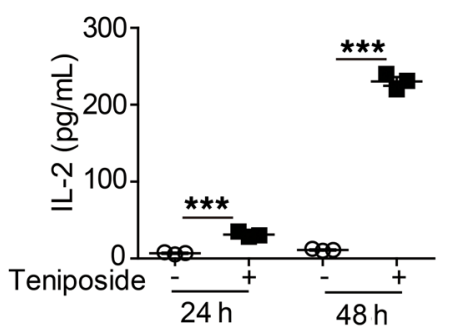

$\mathbf{F}$

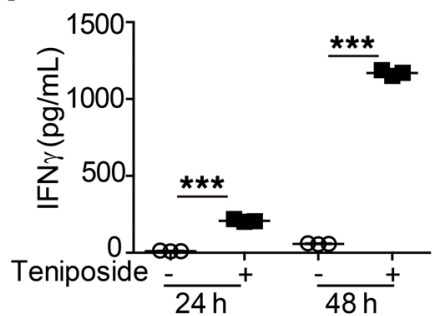

Figure 4. Teniposide-treated tumor cells induced T cell activation and DC maturation. (A-D) B16-OVA cells were treated with teniposide or DMSO for 16 hours, then cocultured with BMDC and B3Z cells for an additional 24 hours, after which B3Z activation was measured by LacZ activity, IL-2 production, and IFN- $\gamma$ production (A-C) and CD69 expression (D). (E-C) B16-OVA cells were treated with teniposide or DMSO for 16 hours, then cocultures with BMDC and OT-I cells for an additional 24 hours or 48 hours, after which OT-I activation was measured by secretion of IL-2 and IFN- $\gamma$ and surface expression of CD69. (H-M) B16-OVA cells were treated with DMSO or indicated concentration of teniposide for 16 hours, then cocultured with BMDCs for an additional 24 hours, after which surface expression of CD80, CD86, CD40, MHC-II, MHC-I, and MHC-I-SIINFEKL on CD11C DCs was determined by FACS. Data in A-M are shown as mean \pm SD of 3 independent experiments. ${ }^{*} P<0.05$; ${ }^{* *} P<0.01 ;{ }^{* *} P<0.001$, unpaired Student's $t$ test $(\mathbf{A}-\mathbf{G}, \mathbf{J}, \mathbf{M}) ; 1$-way ANOVA with Bonferroni's post test $(\mathbf{H}, \mathbf{I}, \mathbf{K}, \mathbf{L})$.

contains the $K$-Ras $G 12 D$ mutation and is known as not sensitive to checkpoint-blockade antibody therapy (31). Mice with established subcutaneous CT26 tumors were treated with teniposide and anti-PD1 antibody alone or in combination. Teniposide treat- ment partially inhibited tumor growth, and teniposide in combination with anti-PD1 achieved the best tumor growth inhibition (Figure 6P). A similar result was observed in the MC38 and PDAC tumor mouse models (Supplemental Figure 7E). Strikingly, when 

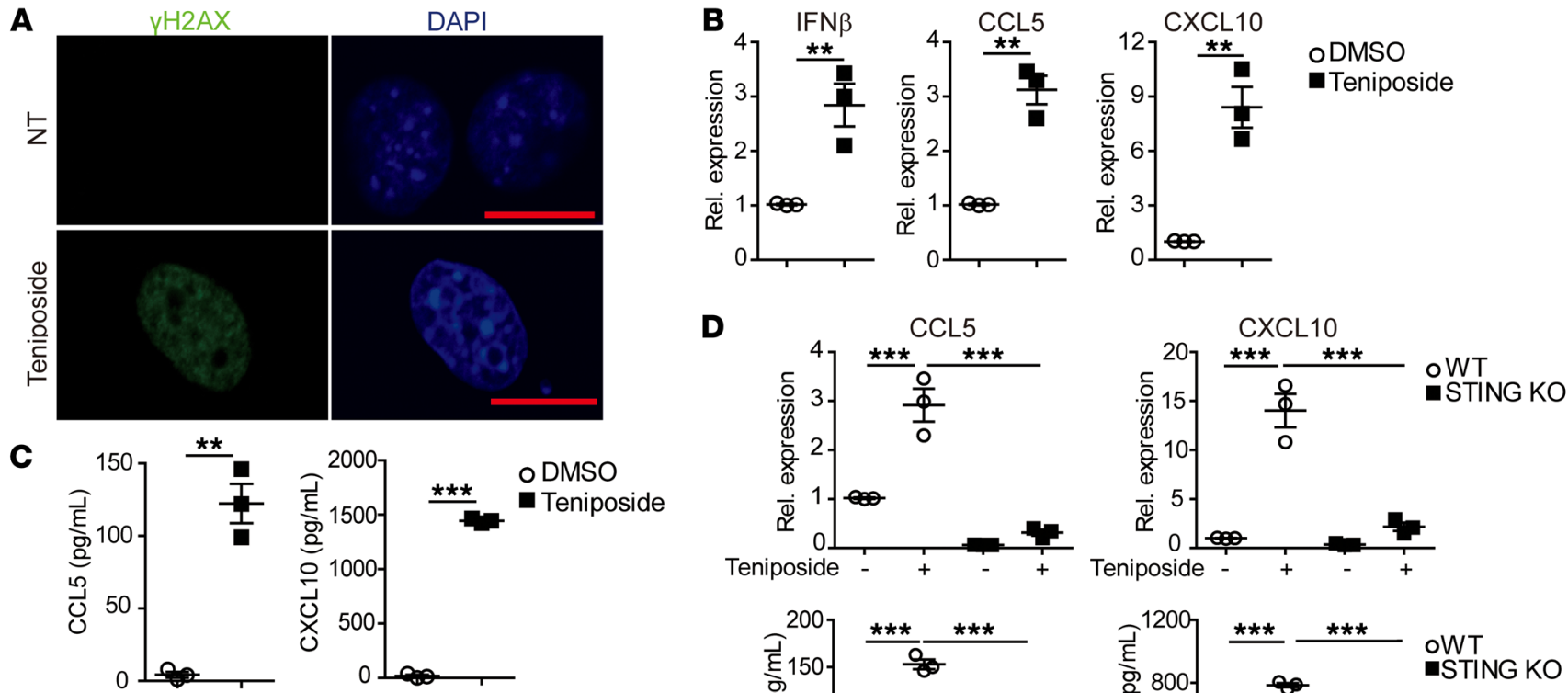

$\mathbf{E}$
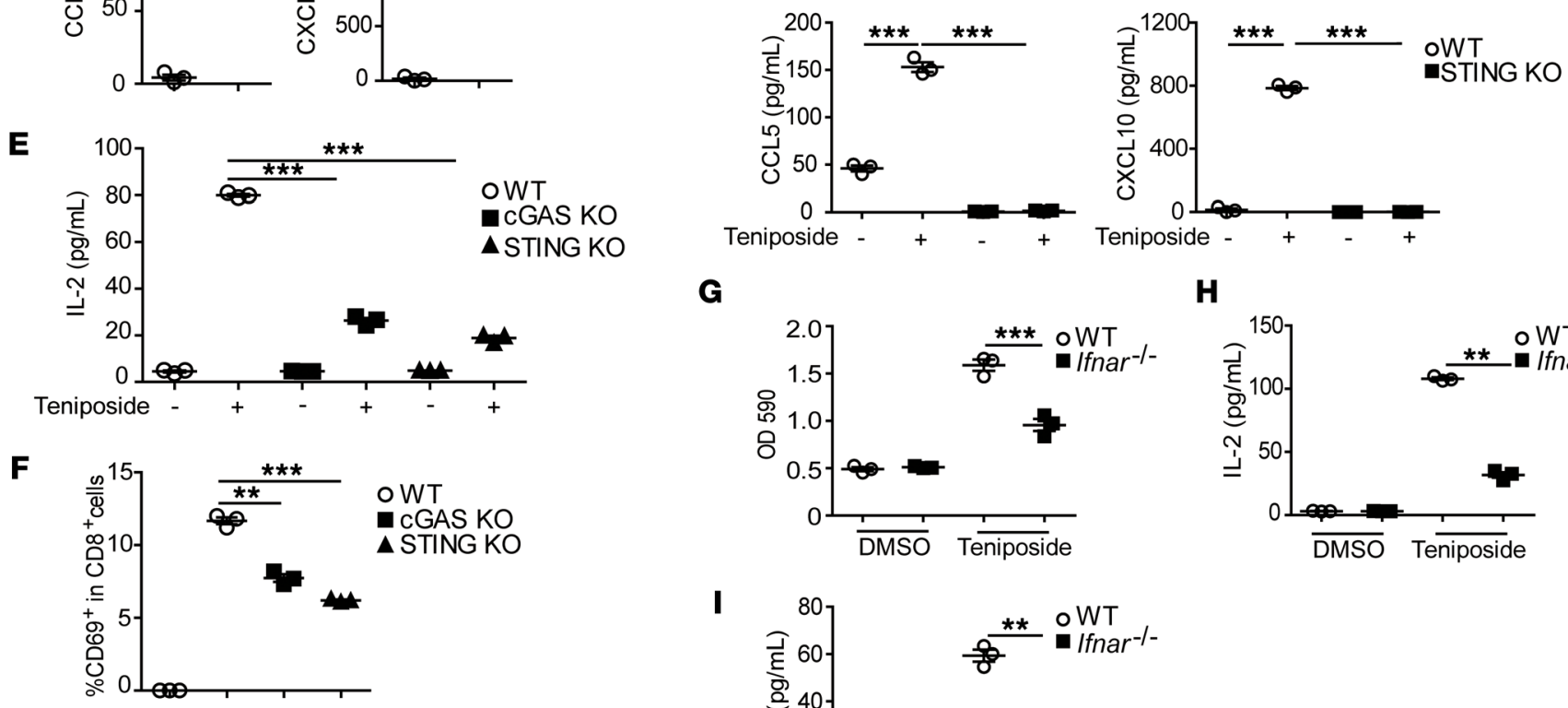

G

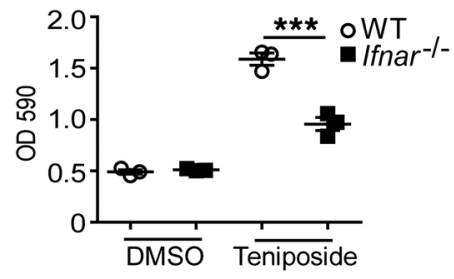

H

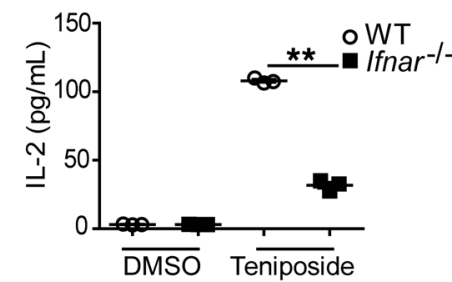

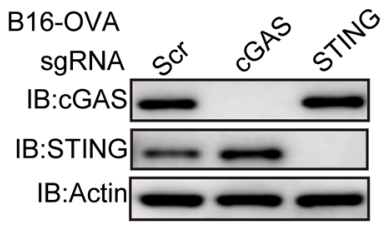

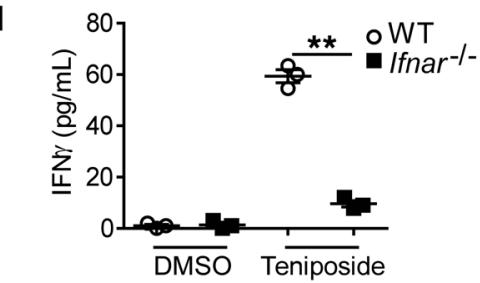

Figure 5. Teniposide activated cGAS/STING-dependent IFN-I signaling in tumor cells. (A) B16 cells were treated with teniposide or DMSO for 24 hours; then $\gamma \mathrm{H} 2 \mathrm{AX}$ expression was detected by immunofluorescence staining. Scale bar: $10 \mu \mathrm{m}$. (B) B16 cells were treated as in $\mathbf{A}$; then the expression levels of IFN- $\beta$, CCL5, and CXCL10 were measured by qPCR. (C) Cells were treated as in $\mathbf{A}$; then the supernatant levels of CCL5 and CXCL10 were measured by ELISA. (D) B16/WT and B16/STING KO cells were treated with teniposide or DMSO for 24 hours; then the levels of mRNA and protein expression of CCL5 and CXLC10 were measured by GPCR and ELISA, respectively. (E and F) B16-OVA/WT, B16-OVA/CGAS-KO and B16-OVA/STING-KO cells were treated with teniposide or DMSO for 16 hours, then cocultured with B3Z+BMDCs for an additional 24 hours. T cell activation was measured by supernatant IL-2 levels and surface expression of CD69. Protein expression of cGAS or STING was measured by Western blot. Actin was used as a loading control. (G-I) B16-OVA cells were treated with teniposide or DMSO for 16 hours, then cocultured with B3Z in the presence of WT or Ifnar ${ }^{-1-}$ BMDCs for an additional 24 hours, after which LacZ activity and the supernatant levels of IL- 2 and IFN- $\gamma$ were determined. Data shown in $\mathbf{A}$ are representative of 1 of 3 independent experiments. Data shown in B-I are represented as mean \pm SD of 3 independent experiment. ${ }^{* *} P<0.01 ;{ }^{* * *} P<0.001$, unpaired Student's $t$ test (B, C, G-I); 1-way ANOVA with Bonferroni's post test (D-F).

STING expression was knocked down by shRNA in CT26 cells, the therapeutic efficacy of teniposide alone or in combination with anti-PD1 was markedly impaired, further supporting the idea that teniposide-induced tumor ICD and antitumor immunity were dependent on tumor-intrinsic STING activation (Figure 6Q and Supplemental Figure 7F). Collectively, these results showed that teniposide could induce immunogenic tumor cell death and activate the immune cells inside the tumor microenvironment, which may pave the way for the enhanced efficacy of anti-PD1 therapy on different tumor types. 
A
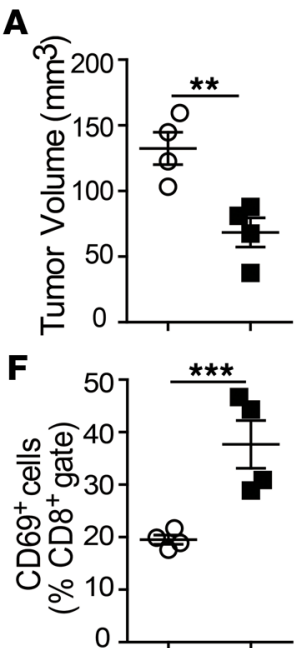

K

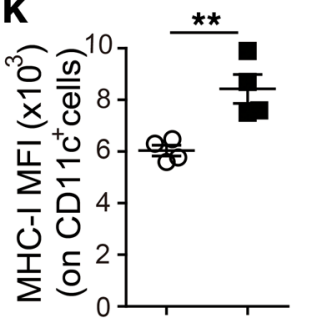

B
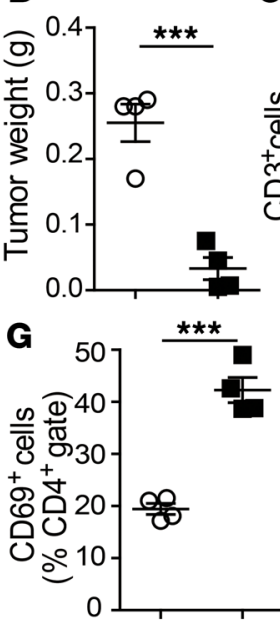

$\mathbf{L}$

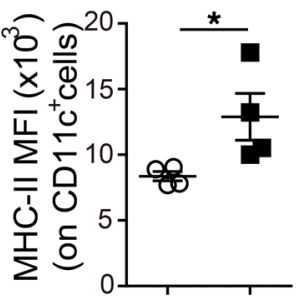

C

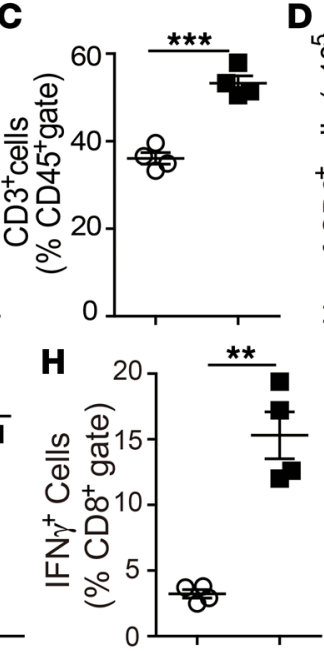

M

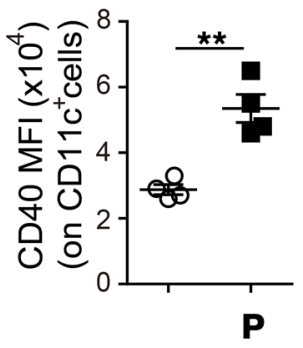

D

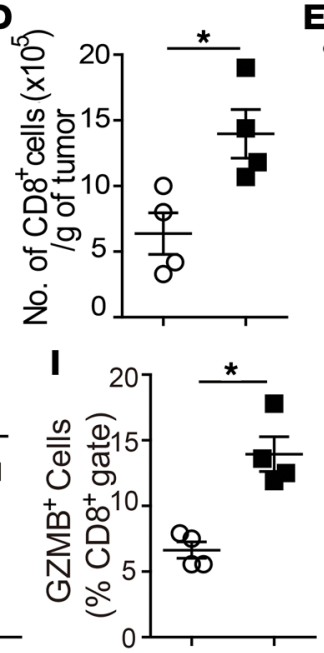

N

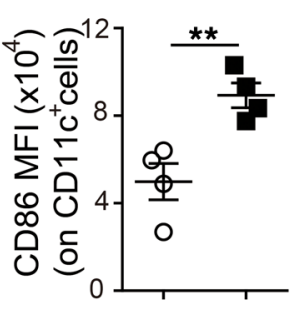

E
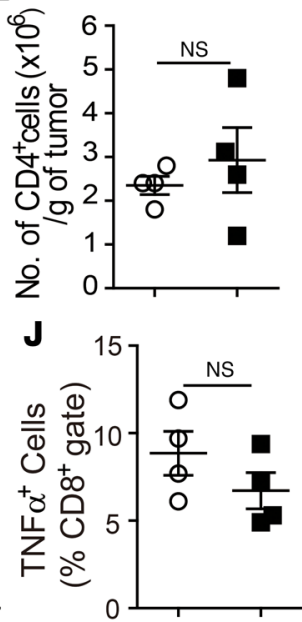

o Vehicle

- Teniposide

\section{0}
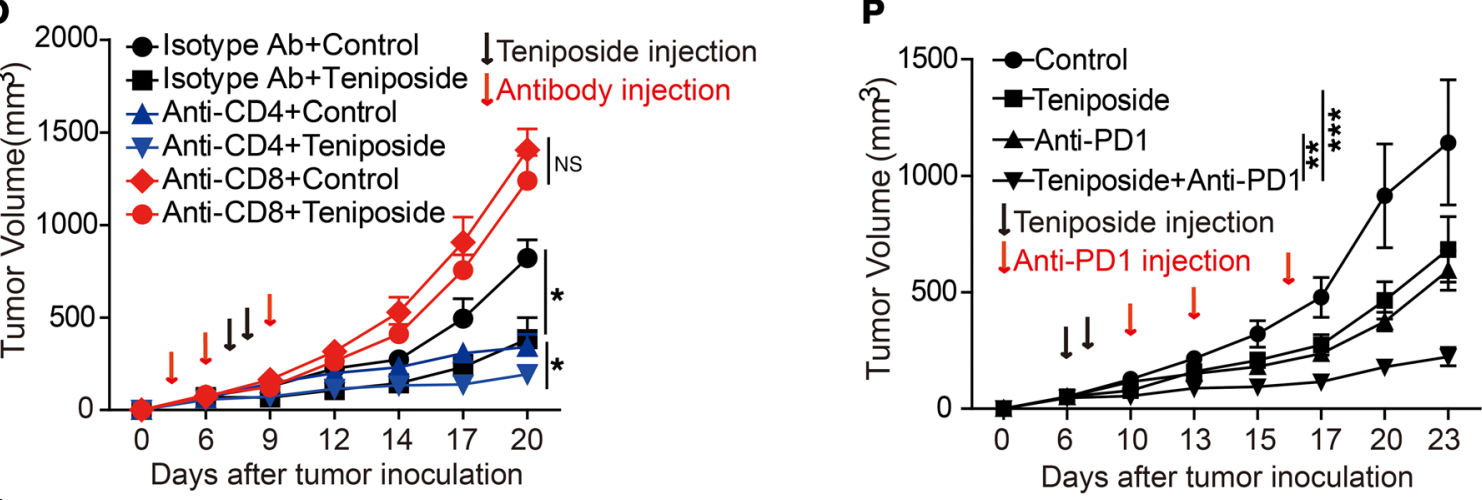

Q
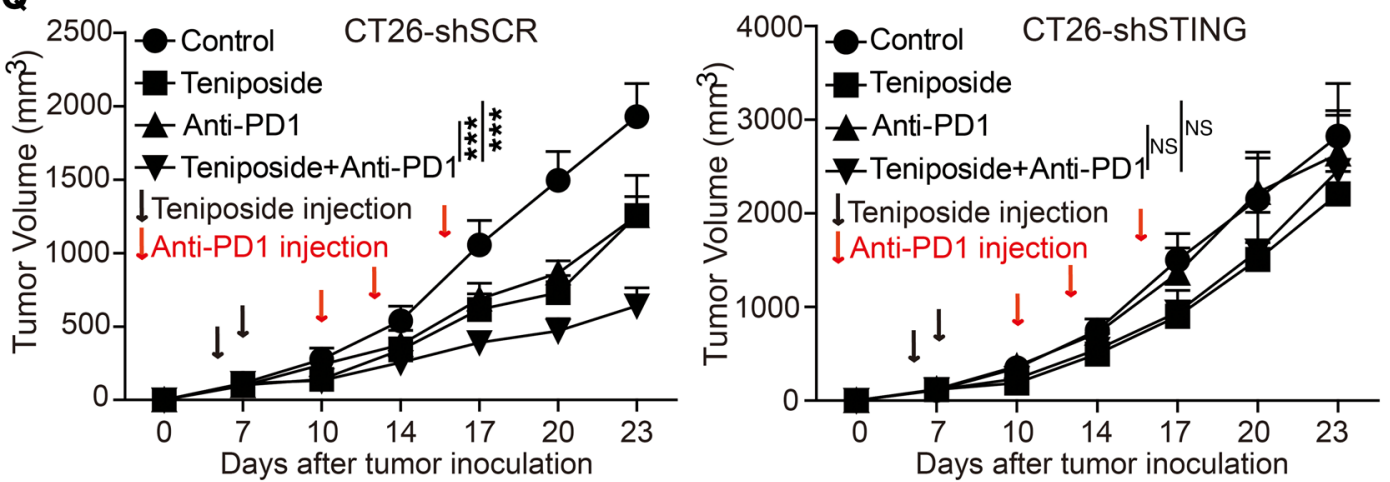

Figure 6. Teniposide induced immune cell infiltration and potentiated efficacy of anti-PD1 therapy in a CT26 mouse tumor model. (A-N) Mice with established CT26 tumors were treated with teniposide or vehicle on days 6 and $7(10 \mathrm{mg} / \mathrm{kg}$, i.p.). Tumors were isolated on day 10 , and tumor-infiltrating immune cells were analyzed by flow cytometry. Data are representative of 1 of 2 independent experiments. Shown are tumor volume (A), tumor weight (B), intratumoral T cells (C), numbers of tumor-infiltrating CD8 ${ }^{+} T$ cells $(\mathbf{D}), C D 4^{+} T$ cells $(\mathbf{E})$, and expression of activation marker CD69 (F and $\left.\mathbf{C}\right)$ and effector molecules IFN- $\gamma$, CZMB, and TNF- $\alpha(\mathbf{H}-\mathrm{J})$ in CD8 ${ }^{+}$T cells. (K-N) Surface expression levels of MHC-I, MHC-II, CD40, and CD86 on CD11c ${ }^{+}$cells were determined by FACS. $n=4$ mice per group. (0) Mice were injected with CD8 or CD4 depletion antibody on days 3,6 , and 9 after CT26 tumor inoculation, followed by teniposide treatment on days 7 and $8(10 \mathrm{mg} / \mathrm{kg}$, i.p.). Tumor volume is shown as mean \pm SD. $n=5$ per group. (P) Mice with established CT26 tumors were treated with teniposide, antiPD1, or teniposide in combination with anti-PD1 at indicated time points. Tumor volume was shown as mean \pm SD. $n=7$ per group. (Q) Mice were inoculated with CT26-shSCR (scramble shRNA as control) or CT26-shSTING cells and then treated with indicated drugs. Tumor volume is shown as mean \pm SD. $n=5$ per group. ${ }^{*} P<0.05$; ${ }^{*} P<0.01$; ${ }^{* *} P<0.001$, unpaired Student's $t$ test $(\mathbf{A}-\mathbf{N})$ or 2 -way ANOVA with Bonferroni's post test $(\mathbf{0}-\mathbf{Q})$. 


\section{Discussion}

The known parameters reflecting ICD include the translocation of CRT, the secretion of ATP, the release of HMGB1, and the recently added IFN-I and CXCL10 $(13,19)$. However, these markers only represent the hallmark changes on tumor cells, but do not directly reflect or guarantee immune activation. Therefor, the gold standard for validating the ICD features of a drug is in vivo vaccination using such drug-treated tumor cells (13). Such in vivo tests generate more reliable results, but are often intensively laborious. Moreover, some of the ICD features rely on the functional capacity of specific intracellular signaling pathways, such as ER stress or the necroptosis pathway, that are required for in vivo tumor immunogenicity $(23,32)$. However, various cancer cell lines may have defects in one or many of these pathways and may not always derive consistent results in testing immunogenicity. Therefore, direct measurement on immune cell activation could circumvent the variation and uncertainty from measurements of markers on tumor cells. In this study, we adapted an antigen-presentation assay to measure $\mathrm{T}$ cell activation induced by drug-treated tumor cells. In combination with a report assay measuring the release of HMGB1, a prototype ICD marker, we identified teniposide, a Top II inhibitor, as a candidate ICD inducer. Indeed, in vivo vaccination experiments validated the capacity of teniposide as a bona fide ICD drug.

Interestingly, a number of Top II inhibitors have been identified as ICD drugs, including mitoxantrone and doxorubicin (18). A liposomal form of irinotecan, a Top I inhibitor, was recently identified as an antitumor drug enhancing efficacy of $\mathrm{T}$ cellbased cancer immunotherapy (33). However, other topoisomerase inhibitors, including camptothecin and etoposide, did not elicit tumor cell ICD in our and others' assays (25). Thus, Top II protein dysfunction per se is unlikely the original trigger of immunogenicity. On the other hand, many chemotherapeutic drugs, including topoisomerase inhibitors, induced DNA damage in tumor cells, but most of them do not elicit tumor ICD, suggesting that DNA damage is insufficient to induce ICD features. Instead, recent findings suggest that the downstream innate immune signaling activation following DNA damage,

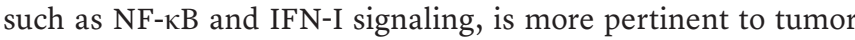
cell immunogenicity $(20,27,28)$.

IFN-I signaling activation has been recently considered as a key feature of ICD (13). It is known that increased IFN-I not only enhances the immunogenicity of tumor cells, such as promoting antigen presentation, but also regulates the tumor microenvironment by recruiting and activating DCs and antitumor T cells (34). The IFN-I pathway expression signature has been linked to positive prognosis in response to chemotherapy $(21,35,36)$. Treatment of various tumor types with the anthracycline class of chemotherapy induces cancer cell-autonomous IFN-I activation, which is dependent on tumor TLR3 and contributes to chemotherapy efficacy (21). Radiation therapy can also induce IFN-I in the tumor microenvironment, which in contrast, is dependent on the cGAS/STING pathway in DCs $(27,37)$. Direct intratumor injection of STING agonists boosted tumor immunogenicity $(38,39)$, and in such a scenario, tumor-intrinsic STING expression seemed less critical, but STING expression in APCs was more important for ensuing antitumor immunity (40). Similarly, oxaliplatin combined with cyclophosphomide boosted tumor immunogenicity through stromal myeloid TLR4 signaling (12). Our results suggest that tumor-intrinsic STING expression is essential for enhanced antitumor therapeutic efficacy when using teniposide in combination with anti-PD1. Consistent with our findings, a recent study reported that a poly ADP ribose polymerase (PARP) inhibitor activated the tumor cell-intrinsic STING pathway to promote DC activation and $\mathrm{T}$ cell recruitment in BRCA1-deficient triple-negative breast cancer tumor models (41). The discrepancy about the role of stroma activation following chemotherapy or irradiation therapy may derive from different treatment regimens or tumor models, but it is most likely that innate immune signaling, including NF- $\mathrm{BB}$ and IFN-I pathway activation, would involve both cancer cell-intrinsic sensitivity and stroma activation, especially when combined with immunotherapy. Our findings highlight an essential role of tumor-intrinsic STING expression for tumor response to chemoimmunotherapy using DNA damaging agents, such as teniposide. As STING expression is often dysregulated in human cancers (42), an immunohistochemical test of intratumoral STING expression may help predict patient response to such combination treatment.

A previous report showed that Top II inhibitors induced IFN-I pathway activation through the ATM and cGAS/STING pathway, which can prevent the Ebola virus infection (43). Consistent with these findings, teniposide-induced IFN-I pathway activation in both tumor cells and tumor tissues depended on the cGAS/STING pathway, as knocked out STING or cGAS blocked IFN-I pathway activation. Intriguingly, a recent work reported that etoposide, another type of Top II inhibitor, elicited NF- $\mathrm{KB}$ activation in a STING-dependent but cGAS-independent fashion (44). In our study, teniposide-induced IFN-I activation required both cGAS and STING in tumor cells. Interestingly, functional cGAS was also essential for tumor immunogenicity recognized by NK cell-mediated antitumor immunity (45). It is possible that etoposide and teniposide induced different signaling pathways that induce DNA damage, or they may have additional unknown targets besides Top II (46). Moreover, we observed that IFNAR receptor deficiency on DCs attenuated the activation of $\mathrm{T}$ cells. This result indicated the essential role of IFN-I in DC activation in antitumor immune response. However, IFNAR deficiency did not completely inhibit $\mathrm{T}$ cell activation, suggesting that other innate immune signaling could also be involved in DC activation and function.

Indeed, we also detected NF- $\mathrm{KB}$ activation in tumor cells following teniposide treatment, and $\mathrm{NF}-\kappa \mathrm{B}$ inhibition in tumor cells by knocking down IKK $\beta$ also attenuated teniposide-induced immunogenicity. NF- $\mathrm{KB}$ activation was required for tumor immunogenicity induced by RIPK1-mediated necroptosis (20). Furthermore, NF- $\kappa \mathrm{B}$ signaling also controlled several types of IFN-I gene expression, such as IFN- $\beta$ (47). Teniposide was therefore identified as being able to induce the activation of NF- $\kappa$ B and cGAS/ STING-mediated IFN-I signaling in tumor cells, both of which contributed to enhanced tumor immunogenicity.

Although both NF-кB and IFN-I signaling have an immunestimulating function, they also induce expression of inhibitory immune molecules, such as PD-L1 $(35,47)$. We found that teniposide induced PD-L1 expression on multiple tumor cells in vitro. In vivo experiments showed that teniposide treatment induced $\mathrm{T}$ cell infiltration and activation in the tumor microenvironment. AntiPD1 treatment has been shown to have superior therapy efficacy 
on multiple tumor types, but the response rate is still much lower than desired. The nonresponder tumors are often found with low intratumor $\mathrm{T}$ cell infiltration or PD-L1 expression and form a cold microenvironment, preventing $\mathrm{T}$ cell reactivation following antiPD1 treatment (3). Therapies that can boost $\mathrm{T}$ cell infiltration or PD-L1 expression inside tumors may have the potential to convert an immune cold tumor to a hot tumor, thereby increasing the tumor response to PD-L1/PD1 blockade and expanding the benefits of anti-PD1 therapy. Recent studies have shown that the CDK inhibitors abemaciclib and dinaciclib can potentiate antitumor immunity and enhance the efficacy of checkpoint blockade $(16,48)$. On the other hand, radiation therapy can also enhance the inhibition of tumor progress by checkpoint blockade therapy, but the effect was limited to specific tumor types $(49,50)$. Here, we demonstrated that teniposide in combination with anti-PD1 resulted in enhanced antitumor efficacy in several mouse tumor models.

Overall, our findings suggest that teniposide could induce both NF-кB activation and cGAS/STING-mediated IFN-I signaling within tumor cells, in turn eliciting tumor immunogenicity and activating tumor microenvironment, which could sensitize tumor response to anti-PD1 treatment. As several clinical trials testing the efficacy of chemoimmunotherapy based on ICD-inducing drugs and checkpoint blockade antibodies are ongoing, our findings provide a potential chemoimmunotherapeutic approach for cancer treatment by using teniposide in combination with anti-PD1 antibody and suggest that a test of intratumoral STING expression may help predict patient response to such chemoimmunotherapy.

\section{Methods}

Mice and reagents. Six- to eight-week-old female C57BL/6J and BALB/c mice were purchased from the Charles River Laboratory. OT-I mice and Ifnar-- mice were obtained from Jackson Laboratory. All the mice were maintained under specific pathogen-free conditions.

The B16 (C57BL/6 mouse melanoma), LSV174T (human colon adenocarcinoma), CT26 (BALB/c mouse colon adenocarcinoma), and HEK293 cell lines were obtained from ATCC. MC38 (C57BL/6 mouse colon adenocarcinoma) was provided by Yang Xuanming (Shanghai Jiaotong University, Shanghai, China). B16-OVA cells were constructed by stably expressing OVA cDNA on B16 cells. PDAC murine pancreatic cancer cells were derived from spontaneous pancreatic cancer tissues of $K$-ras (G12D); $p 53^{-/-}$mice (51). DC2.4, a murine DC line, was provided by Kenneth Dock (University of Massachusetts Medical School, Worcester, Massachusetts, USA). B3Z hybridoma cells were provided by Nilabh Shastri (University of California, Berkeley, Berkeley, California, USA). All cell lines were tested and found mycoplasma free. The cells were maintained either with DMEM (Invitrogen) supplemented with 10\% FBS and 1\% penicillin-streptomycin or RPMI 1640 (Invitrogen) supplemented with $1 \%$ penicillin-streptomycin and $10 \% \mathrm{FBS}$ in a humidified atmosphere at $37^{\circ} \mathrm{C}$ and $5 \% \mathrm{CO}_{2}$.

For primary cell cultures, single-cell suspensions of mouse bone marrow cells were cultured in RPMI-1640 medium containing 10\% FBS, supplemented with $20 \mathrm{ng} / \mathrm{ml} \mathrm{GM-CSF}$ and IL-4 (Peprotech, 31503, 214-14). The culture media was refreshed every 2 days. DMSO was from MilliporeSigma (D2650); TNF- $\alpha$ was from Peprotech (315-01A); birinapant (A4219), LCL161 (A3541), z-VAD-FMK (A1902), mitoxantrone (B2114), teniposide (A8532), etoposide (A1971), doxorubicin (A3966), daunorubicin (B1099), nec-1 (A4213), SP600125 (A4604),
NAC (A8356), and Bay 117082 (A4210) were all purchased from Apexbio Inc. Anti-mouse PD1 antibody (clone G4) was provided by Lieping Chen (Yale University, New Haven, Connecticut, USA) (52).

LacZ activity measurement. The procedures for lacZ activity measurement were performed according to previously described protocols (53). Briefly, after activation, B3Z cells in the wells of a cell-culture plate were lysed and freeze-thawed and then added to $50 \mu \mathrm{L} /$ well PBS containing $0.5 \%$ BSA and $100 \mu \mathrm{L} /$ well substrate solution $(1 \mathrm{mg} / \mathrm{mL}$ chlorophenolred $\beta$-D-galactopyranoside) dissolved in $\beta$-galactosidase buffer. The plate was incubated at $37^{\circ} \mathrm{C}$ for 12 to 18 hours until color development reached a proper level, followed by color intensity reading at $590 \mathrm{~nm}$ using a microtiter plate reader.

Gluc measurement. HMGB1-Gluc reporter was stably transfected into tumor cells by a lentiviral-based backbone, and the stably transfected cells were treated with drugs for indicated time points. A $50 \mu \mathrm{L}$ culture medium was collected from each sample to measure luciferase activity by using the Renilla Luciferase Assay (PromegaE2820) according to the manufacturer's instructions (54).

Detection of genomic DNA in cytosolic extracts. The procedure for genomic DNA detection in cytoplasm was performed as previously described (55). Cytosolic DNA was extracted and quantified via quantitative PCR (qPCR) using the primer specific for genomic DNA (Polg1). The primer sequence of polg1 was as follows: forward primer, 5'-GATGAATGGGCCTACCTTGA-3', and reverse primer, 5'-TGGGGTCCTGTTTCTACAGC-3'.

CRISPR/Cas9 KO and shRNA knockdown. STING-deficient and cGAS-deficient cells were constructed through the CRISPR/Cas9 system (56). The sgRNA sequences were designed using the Optimized CRISPR Design (http://chopchop.cbu.uib.no/). The guide sequences used were $5^{\prime}$-GACGCAAAGATATCTCGGAGG-3' for cGAS and 5'-GTACCTTGGTAGACAATGAGG-3' for STING. The sgRNA was inserted into the LentiCRISPR v2 vector, which also contained the Streptococcus pyogenes Cas9 nuclease gene. The cells were transiently transfected with plasmids, followed by selection with puromycin for 2 days, and then the KO effect was confirmed by Western blot analysis of whole-cell protein extracts.

Expression of IKK $\beta$, TBK1, and IRF3 was knocked down by indicated shRNA in tumor cells. Briefly, shRNA lentiviral vectors were cotransfected with pspax2 and pMD2.G packaging plasmids in 293T cells. The supernatants were harvested 48 hours after transfection and used for infection with tumor cells, followed by puromycin selection for 2 days. The knockdown effect was assessed by Western blot analysis of whole-cell protein extracts.

Western blot, immunofluorescence, and immunohistochemistry. The procedures for protein sample preparation from cell cultures, protein quantification, Western blot, and data analyses were performed as previously described (57). The following antibodies were used for Western blot analyses: Gaussia (NEB, catalog E8023), actin (MilliporeSigma, catalog A3854), cGAS (CST, catalog 31659), STING (CST, catalog 13647), STAT1 (CST, catalog 14994), p-STAT1 (CST, catalog 9167), p-p65 (CST, catalog 3036), p65 (CST, catalog 8248), TBK1 (CST, catalog 3504), p-IRF3 (CST, catalog 29047), and IRF3 (CST, catalog 4302). Protein bands were visualized by chemiluminescence using an ECL detection kit (Thermo Scientific, 32106).

For immunofluorescence, the cells were fixed in $4 \%$ paraformaldehyde (PFA) in PBS for 20 minutes at room temperature (RT), washed twice with PBS, and permeabilized with $0.5 \%$ Triton X-100 in 
PBS for 10 minutes. After 2 additional washes, the cells were blocked with 2\% BSA and 2\% FBS in PBS (IFF) for 1 hour at RT. The cells were then incubated with $\gamma \mathrm{H} 2 \mathrm{AX}$ antibody (CST, 9718) in IFF at $4^{\circ} \mathrm{C}$ overnight. They were then washed 3 times with PBS, each for 10 minutes, followed by incubation with FITC-conjugated secondary antibodies (CST, 4412) and $1 \mu \mathrm{g} / \mathrm{ml}$ of DAPI in IFF for 1 hour at RT. After that, the cells were washed again 3 times with PBS, and the slides were examined using fluorescent microscopy.

For immunohistochemistry, the tissue sections were deparaffinized in xylene rehydrated by incubation in serial ethanol baths (95\%$30 \%, 2$ minutes per bath). Epitope retrieval was performed through incubation in $10 \mathrm{mM}$ citrate buffer $(\mathrm{pH}=6.0)$ for 30 to 40 minutes. Endogenous peroxidase activity was inhibited by treatment with $3 \%$ $\mathrm{H}_{2} \mathrm{O}_{2}$ for 10 minutes. The tissue slides were then incubated overnight at $4^{\circ} \mathrm{C}$ with anti-CD8 (dilution: 1:200, CST, catalog 70306) or antiCRT (dilution: 1:100, Abcam, catalog ab2907) primary antibodies. After washes in PBS, the slides were incubated for 30 minutes at RT with a secondary antibody (Dako), and the signal was subsequently detected by the chromogenic substrate (Dako).

Detection of apoptosis, surface CRT staining, and LDH assay. Tumor cell lines were seeded in 24-well multiple plates, then treated with teniposide or DMSO at indicated time points. Tenipside-induced tumor cell death was assessed using the Annexin V-Propidium Iodide Apoptosis Detection Kit (BD, 556547), and detection of surface CRT (Abcam, ab2907) and measurement of LDH release used the CytoTox96 Non-Radioactive Assay Kit (Promega, G1780). The procedures were performed following the manufacturers' instructions. Briefly, LDH is a cytosolic soluble enzyme and will leak into culture medium when cells undergo cell death. Afterwards, the enzyme activity in the medium could be quantified by a colorimetrical assay.

$T$ cell and DC activation assay. B16-OVA tumor cells were treated with teniposide for 16 hours. Treated tumor cells were then cocultured with DCs and T cells (B3Z or OT-I cells) for additional indicated time points. LacZ activity assay was performed as previously described. Supernatant levels of IL-2 and IFN- $\gamma$ were measured by ELISA kits (eBioscience, 88-7024-88, 88-7314-22). T cells were stained with fluorescence-labeled antibodies against CD8 (eBioscience, 11-008182), CD69 (BioLegend, 104514), IFN- $\gamma$ (eBioscience, 25-7311-82), and GZMB (eBioscience, 48-8898-82). DCs were stained with CD11c (eBioscience, 61-0114-82), MHC-II (eBioscience, 11-5321-82), CD40 (eBioscience, 12-0401-82), CD86 (eBioscience, 12-0862-82), CD80 (eBioscience, 46-0801-82), MHC-I (eBioscience, 48-5999-82), and MHC-I SIINFEKL (eBioscience, 17-5743-80). After antibody staining, cells were then analyzed using flow cytometry.

Real-time PCR and ELISA analysis. Total RNA was isolated using TRIzol (Invitrogen, 15596018) according to the manufacturer's instructions. RNA was reverse transcribed using the PrimeScript Reverse Transcriptase Reagent KitwithgDNAEraser(Takara, RR036A). Real-timePCR was performed using the SYBR Premix Kit (Genstar, A301) and analyzed using the Bio-Rad CFX96 thermal cycler. The primer sequences used for the investigated mouse genes were as follows: actin, forward: 5'-AGAGGGAAATCGTGCGTGAC-3', actin, reverse: 5'-CAATAGTGATGACCTGGCCGT; CCL5, forward: GCTGCTTTGCCTACCTCTCC-3', CCL5, reverse: 5'-TCGAGTGACAAACACGACTGC-3'; CXCL10, forward: 5'-CCAAGTGCTGCCGTCATTTTC-3', CXCL10, reverse: 5'-GGCTCGCAGGGATGATTTCAA-3'; IFN- $\beta$, forward: 5'-CAGCTCCAAGAAAGGACGAA-3', IFN- $\beta$, reverse: 5'-GGCAGTGTAACTCTTCTGCAT-3'.
Supernatant levels of CCL5 and CXCL10 were measured by ELISA kits (R\&D Systems, DY478, DY466) following the manufacturer's instructions.

Tumor growth and treatments and analytics. For the immunization study, $3 \times 10^{6}$ CT26 cells, either freeze-thawed 3 times in liquid nitrogen or treated with $50 \mu \mathrm{M}$ teniposide or $50 \mu \mathrm{M}$ etoposide, were inoculated subcutaneously into the lower left flank of BALB/c mice. Eight days later, $5 \times 10^{5}$ live CT26 cells were inoculated into the right flank, and the tumor growth was monitored. For immunophenotyping analysis of tumor microenvironment, CT26 $\left(5 \times 10^{5}\right.$ cells $)$ or B16 $\left(1 \times 10^{6}\right.$ cells $)$ tumor cells were subcutaneously injected into the flank of BALB/c or B6 mice. Tumors were allowed to grow for 6 or 7 days, and teniposide (dissolved in 10\% Cremophor EL in PBS, MilliporeSigma) or vehicle was administered by i.p. injection $(10 \mathrm{mg} / \mathrm{kg})$ twice at indicated time points. For analysis of immune cell populations, mouse tumors were dissociated by gentleMACS (Miltenyi Biotec) and filtered through $70 \mu \mathrm{m}$ cell strainers to generate single-cell suspensions, then stained with CD45 (eBioscience, catalog 48-0451-82), CD3 (eBioscience, catalog 46-0031-82), CD4 (eBioscience, catalog 47-0041-82), CD8 (eBioscience, catalog 11-0081-82), CD69 (BioLegend, catalog 104514), IFN- $\gamma$ (eBioscience, catalog 25-7311-82), GZMB (eBioscience, catalog 12-8898-82), and TNF- $\alpha$ (eBiosicence, catalog 17-7321-82) for $\mathrm{T}$ cell analysis. For DC analysis, cells were stained with CD11c (eBioscience, catalog 61-0114-82), MHC-II (eBioscience, catalog 47-5321-82), CD86 (eBioscience, catalog 11-0862-82), CD40 (eBioscience, catalog 12-0401-82), and MHC-I (eBioscience, catalog 48-5999-82). Fluorescence data were acquired on a BD LSR Fortessa Cytometer and analyzed using FlowJo, version 7.6.5. For in vivo study, CT26 $\left(5 \times 10^{5}\right.$ cells $)$, MC38 $\left(1 \times 10^{6}\right.$ cells $)$, or PDAC $\left(1 \times 10^{6}\right.$ cells $)$ tumor cells were subcutaneously injected into the flank of BALB/c or B6 mice. The growth of the tumors was observed for 6 to 7 days, after which teniposide or vehicle was administered by i.p. injection $(10 \mathrm{mg} /$ $\mathrm{kg}$ ) twice at indicated time points, followed by 3 i.p. injections of antiPD1 $(100 \mu \mathrm{g} / \mathrm{mouse}$, once every 3 days). Tumor volume was calculated as $0.5 \times$ tumor length $\times(\text { tumor width })^{2}$, where the longer dimension was considered as tumor length.

Anti-CD4 (catalog BE0003-1), anti-CD8 (catalog BE0004-1), and isotype (catalog BE0089) depletion antibodies were purchased from Bio X Cell. Depletion antibodies were i.p. injected on days 3, 6, and $9(100 \mu \mathrm{g} / \mathrm{mice})$, after tumor cells were inoculated, and depletion effect was confirmed by flow cytometry.

Statistics. Data were analyzed using GraphPad Prism 5 (GraphPad Software). Comparisons between 2 groups were analyzed using a 2-tailed unpaired Student's $t$ test. Comparisons between multiple groups were analyzed using 1-way ANOVA with Bonferroni's post test or 2-way ANOVA with Bonferroni's post test for tumor growth study. Statistical significance was defined as a $P$ value of less than 0.05 .

Study approval. All mice were maintained under specific pathogen-free conditions and in accordance with the animal experimental guidelines of Sun Yat-sen University. All animal procedures were approved by the Institutional Animal Care and Use Committee of Sun Yat-sen University.

\section{Author contributions}

ZW and XX conceived and designed the study. ZW conducted most experiments and wrote the manuscript. JC, HZ, FX, WH, $\mathrm{XW}, \mathrm{JH}, \mathrm{ML}$, and $\mathrm{W} \mathrm{Lu}$ performed parts of the experiments. 
GZ, PZ, PH, LPX, SC, and W Li provided reagents and analyzed data. LPX and XX supervised the project and contributed to writing the manuscript.

\section{Acknowledgments}

We thank Cliff Y. Yang and Junchao Dong for critical reading of the manuscript, Jibin Li for statistical assistance, and Seeruttun Sharvesh Raj for language editing. This work was supported by grants from the National Key R\&D Program of China (2018YFC1313300/2018YFC1313304 and 2017YFC0908503), the National Natural Science Foundation of China (81472578, 81773051, 81803005, 81572409), the China Postdoctoral Science Foundation (2017M622884), the Guangdong Innovative and Entrepreneurial Research Team Program (2016ZT06S638), the Science and Technology Project of Guangdong Province (2017A020215031), and the Guangdong Esophageal Cancer Institute (M201607).

Address correspondence to: Xiaojun Xia or Liang-ping Xia, State Key Laboratory of Oncology in South China, Sun Yat-sen University Cancer Center, 651 Dongfengdong Road, Guangzhou 510060, China. Phone: 86.20.87343997; Email: xiaxj@sysucc.org.cn (XX); xialp@sysucc.org.cn (LPX).
1. Topalian SL, et al. Immunotherapy: The path to win the war on cancer? Cell. 2015;161(2):185-186.

2. Galluzzi L, Chan TA, Kroemer G, Wolchok JD, López-Soto A. The hallmarks of successful anticancer immunotherapy. Sci Transl Med. 2018;10(459):eaat7807.

3. Zou W, Wolchok JD, Chen L. PD-L1 (B7-H1) and $\mathrm{PD}-1$ pathway blockade for cancer therapy: Mechanisms, response biomarkers, and combinations. Sci Transl Med. 2016;8(328):328rv4.

4. Yan L, Zhang W. Precision medicine becomes reality-tumor type-agnostic therapy. Cancer Commun (Lond). 2018;38(1):6.

5. Ansell SM, et al. PD-1 blockade with nivolumab in relapsed or refractory Hodgkin's lymphoma. N Engl J Med. 2015;372(4):311-319.

6. Garon EB, et al. Pembrolizumab for the treatment of non-small-cell lung cancer. $N$ Engl J Med. 2015;372(21):2018-2028.

7. Chen L, Han X. Anti-PD-1/PD-L1 therapy of human cancer: past, present, and future. J Clin Invest. 2015;125(9):3384-3391.

8. Rizvi NA, et al. Cancer immunology. Mutational landscape determines sensitivity to PD-1 blockade in non-small cell lung cancer. Science. 2015;348(6230):124-128.

9. Ayers M, et al. IFN- $\gamma$-related mRNA profile predicts clinical response to PD-1 blockade. JClin Invest. 2017;127(8):2930-2940.

10. Turajlic S, et al. Insertion-and-deletion-derived tumour-specific neoantigens and the immunogenic phenotype: a pan-cancer analysis. Lancet Oncol. 2017;18(8):1009-1021.

11. Overman MJ, et al. Durable clinical benefit with nivolumab plus ipilimumab in DNA mismatch repair-deficient/microsatellite instability-high metastatic colorectal cancer. J Clin Oncol. 2018;36(8):773-779.

12. Pfirschke $C$, et al. Immunogenic chemotherapy sensitizes tumors to checkpoint blockade therapy. Immunity. 2016;44(2):343-354.

13. Galluzzi L, Buqué A, Kepp O, Zitvogel L, Kroemer G. Immunogenic cell death in cancer and infectious disease. Nat Rev Immunol. 2017;17(2):97-111.

14. Casares N, et al. Caspase-dependent immunogenicity of doxorubicin-induced tumor cell death. JExp Med. 2005;202(12):1691-1701.

15. Mattarollo SR, Loi S, Duret H, Ma Y, Zitvogel L, Smyth MJ. Pivotal role of innate and adaptive immunity in anthracycline chemotherapy of established tumors. Cancer Res. 2011;71(14):4809-4820.

16. Hossain DMS, et al. Dinaciclib induces immunogen- ic cell death and enhances anti-PD1-mediated tumor suppression. JClin Invest. 2018;128(2):644-654.

17. Menger L, et al. Cardiac glycosides exert anticancer effects by inducing immunogenic cell death. Sci Transl Med. 2012;4(143):143ra99.

18. Obeid M, et al. Calreticulin exposure dictates the immunogenicity of cancer cell death. Nat Med. 2007;13(1):54-61.

19. Michaud M, et al. Autophagy-dependent anticancer immune responses induced by chemotherapeutic agents in mice. Science. 2011;334(6062):1573-1577.

20. Yatim N, et al. RIPK1 and NF- $\kappa \mathrm{B}$ signaling in dying cells determines cross-priming of $\mathrm{CD}^{+} \mathrm{T}$ cells. Science. 2015;350(6258):328-334.

21. Sistigu A, et al. Cancer cell-autonomous contribution of type I interferon signaling to the efficacy of chemotherapy. Nat Med. 2014;20(11):1301-1309.

22. Zappasodi R, Merghoub T, Wolchok JD. Emerging concepts for immune checkpoint blockade-based combination therapies. Cancer Cell. 2018;34(4):690.

23. Yang $\mathrm{H}$, et al. Contribution of RIP3 and MLKL to immunogenic cell death signaling in cancer chemotherapy. Oncoimmunology. 2016;5(6):e1149673.

24. Ma Y, Pitt JM, Li Q, Yang H. The renaissance of anti-neoplastic immunity from tumor cell demise. Immunol Rev. 2017;280(1):194-206.

25. Sukkurwala AQ, et al. Screening of novel immunogenic cell death inducers within the NCI Mechanistic Diversity Set. Oncoimmunology. 2014;3:e28473.

26. Kepp O, et al. Consensus guidelines for the detec tion of immunogenic cell death. Oncoimmunology. 2014;3(9):e955691.

27. Deng L, et al. STING-dependent cytosolic DNA sensing promotes radiation-induced type I interferon-dependent antitumor immunity in immunogenic tumors. Immunity. 2014;41(5):843-852.

28. Woo SR, et al. STING-dependent cytosolic DNA sensing mediates innate immune recognition of immunogenic tumors. Immunity. 2014;41(5):830-842.

29. Dou Z, et al. Cytoplasmic chromatin triggers inflammation in senescence and cancer. Nature. 2017;550(7676):402-406.

30. Glück S, et al. Innate immune sensing of cytosolic chromatin fragments through cGAS promotes senescence. Nat Cell Biol. 2017;19(9):1061-1070.

31. Ebert PJR, et al. MAP kinase inhibition promotes $\mathrm{T}$ cell and anti-tumor activity in combination with PD-L1 checkpoint blockade. Immunity.
2016;44(3):609-621.

32. Martins I, et al. Restoration of the immunogenicity of cisplatin-induced cancer cell death by endoplasmic reticulum stress. Oncogene. 2011;30(10):1147-1158.

33. McKenzie JA, et al. The effect of topoisomerase I Inhibitors on the efficacy of T-cell-based cancer immunotherapy. J Natl Cancer Inst. 2018;110(7):777-786.

34. Woo SR, Corrales L, Gajewski TF. Innate immune recognition of cancer. Annu Rev Immunol. 2015;33:445-474

35. Zitvogel L, Galluzzi L, Kepp O, Smyth MJ, Kroemer G. Type I interferons in anticancer immunity. Nat Rev Immunol. 2015;15(7):405-414.

36. Bald T, et al. Immune cell-poor melanomas benefit from PD-1 blockade after targeted type I IFN activation. Cancer Discov. 2014;4(6):674-687.

37. Burnette BC, et al. The efficacy of radiotherapy relies upon induction of type i interferon-dependent innate and adaptive immunity. Cancer Res. 2011;71(7):2488-2496.

38. Fu J, et al. STING agonist formulated cancer vaccines can cure established tumors resistant to PD-1 blockade. Sci Transl Med. 2015;7(283):283ra52.

39. Corrales L, et al. Direct activation of STING in the tumor microenvironment leads to potent and systemic tumor regression and immunity. Cell Rep. 2015;11(7):1018-1030.

40. Ahn J, Xia T, Rabasa Capote A, Betancourt D, Barber GN. Extrinsic phagocyte-dependent STING signaling dictates the immunogenicity of dying cells. Cancer Cell. 2018;33(5):862-873.e5.

41. Pantelidou $\mathrm{C}$, et al. PARP inhibitor efficacy depends on $\mathrm{CD}^{+} \mathrm{T}$-cell recruitment via intratumoral STING pathway activation in BRCAdeficient models of triple-negative breast cancer. Cancer Discov. 2019;9(6):722-737.

42. Xia T, Konno H, Ahn J, Barber GN. Deregulation of STING signaling in colorectal carcinoma constrains DNA damage responses and correlates with tumorigenesis. Cell Rep. 2016;14(2):282-297.

43. Luthra P, et al. Topoisomerase II inhibitors induce DNA damage-dependent interferon responses circumventing Ebola virus immune evasion. MBio. 2017;8(2):e00368-17.

44. Dunphy G, et al. Non-canonical activation of the DNA sensing adaptor STING by ATM and IFI16 mediates NF- $\mathrm{B}$ signaling after nuclear DNA damage. Mol Cell. 2018;71(5):745-760.e5.

45. Marcus A, Mao AJ, Lensink-Vasan M, Wang L, Vance RE, Raulet DH. Tumor-derived cGAMP 
triggers a STING-mediated interferon response in non-tumor cells to activate the NK cell response. Immunity. 2018;49(4):754-763.e4.

46. Pommier Y. Drugging topoisomerases: lessons and challenges. ACS Chem Biol. 2013;8(1):82-95.

47. Taniguchi K, Karin M. NF-кB, inflammation, immunity and cancer: coming of age. Nat Rev Immunol. 2018;18(5):309-324.

48. Goel S, et al. CDK4/6 inhibition triggers anti-tumour immunity. Nature. 2017;548(7668):471-475.

49. Shaverdian N, et al. Previous radiotherapy and the clinical activity and toxicity of pembrolizum$\mathrm{ab}$ in the treatment of non-small-cell lung cancer: a secondary analysis of the KEYNOTE-001 phase 1 trial. Lancet Oncol. 2017;18(7):895-903.
50. Ko EC, Raben D, Formenti SC. The integration of radiotherapy with immunotherapy for the treatment of non-small cell lung cancer. Clin Cancer Res. 2018;24(23):5792-5806.

51. Ling J, et al. KrasG12D-induced IKK2/ $\beta /$ NF- $\mathrm{KB}$ activation by IL-1 $\alpha$ and $\mathrm{p} 62$ feedforward loops is required for development of pancreatic ductal adenocarcinoma. Cancer Cell. 2012;21(1):105-120.

52. Hirano F, et al. Blockade of B7-H1 and PD-1 by monoclonal antibodies potentiates cancer therapeutic immunity. Cancer Res. 2005;65(3):1089-1096.

53. Lee YR, et al. Cyclosporin A and tacrolimus, but not rapamycin, inhibit MHC-restricted antigen presentation pathways in dendritic cells. Blood. 2005;105(10):3951-3955.
54. Wang Z, Ji J, Peng D, Ma F, Cheng G, Qin FX. Complex regulation pattern of IRF3 activation revealed by a novel dimerization reporter system. JImmunol. 2016;196(10):4322-4330.

55. West AP, et al. Mitochondrial DNA stress primes the antiviral innate immune response. Nature. 2015;520(7548):553-557.

56. Sanjana NE, Shalem O, Zhang F. Improved vectors and genome-wide libraries for CRISPR screening. Nat Methods. 2014;11(8):783-784.

57. Peng D, Wang Z, Huang A, Zhao Y, Qin FX. A novel function of F-box protein FBXO17 in negative regulation of type I IFN signaling by recruiting PP2A for IFN regulatory factor 3 deactivation. J Immunol. 2017;198(2):808-819. 